\title{
Blast-wave driven Kelvin-Helmholtz shear layers in a laser driven high-energy-density plasma
}

\author{
O.A. Hurricane - J.F. Hansen - E.C. Harding • V.A. Smalyuk • B.A. Remington • \\ G. Langstaff · H.-S. Park · H.F. Robey · C.C. Kuranz • M.J. Grosskopf · R.S. Gillespie
}

Received: 6 May 2010 / Accepted: 13 December 2010 / Published online: 29 December 2010

(c) The Author(s) 2010. This article is published with open access at Springerlink.com

\begin{abstract}
The first successful high energy density KelvinHelmholtz $(\mathrm{KH})$ shear layer experiments (O.A. Hurricane et al. in Phys. Plasmas, 16:056305, 2009; E.C. Harding et al. in Phys. Rev. Lett., 103:045005, 2009) demonstrated the ability to design and field a target that produces, in a controlled fashion, an array of large diagnosable $\mathrm{KH}$ vortices. Data from these experiments vividly showed the complete evolution of large $(\sim 400 \mu \mathrm{m})$ distinct eddies, from formation to apparent turbulent break-up in the span of about 75 ns. A second set of experiments, in which the density of a key carbon-foam material was varied, was recently performed. The new series showed a great deal of fine-structure that was not as apparent as in the original experiments. In this paper, the results of both experiments will be discussed along with supporting theory and simulation. An attempt is made to connect these observations with some turbulent scale-lengths. Finally, we speculate about the possible connection of these experiments to astrophysical contexts.
\end{abstract}

Keywords Kelvin-Helmholtz instability · Shear layer . Vortex

O.A. Hurricane $(\bowtie) \cdot$ J.F. Hansen · V.A. Smalyuk

B.A. Remington - G. Langstaff · H.-S. Park · H.F. Robey

Lawrence Livermore National Laboratory, 7000 East Avenue,

Livermore, CA 94550, USA

e-mail: hurricane1@1lnl.gov

\section{J.F. Hansen}

General Atomics, 3550 General Atomics Court, San Diego,

CA 92121-1122, USA

E.C. Harding - C.C. Kuranz · M.J. Grosskopf · R.S. Gillespie

Department of Atmospheric, Oceanic, and Space Sciences, University of Michigan, 2455 Hayward St., Ann Arbor, MI 48109-2143, USA

\section{Introduction}

In May 2008, our team fielded the first successful highenergy-density-plasma (HEDP) Kelvin-Helmholtz (KH) experiments (Hurricane et al. 2009; Harding et al. 2009) on the Omega laser at the University of Rochester. These experiments demonstrated the conceptual design (Hurricane 2008) that relied upon shock driven baroclinic vorticity production and also showed that vivid high quality data (see Fig. 1) could be obtained on KH in a HEDP environment.

The basic configuration consists of a stack of opaque high density plastic and low density foam all of which is contained in a shock tube of rectangular cross-section, made from $\mathrm{Be}$ so as to be able to radiograph through it with $\mathrm{x}$ rays of a few keV energy (see Fig. 2) - details of the target design can be found in Hurricane et al. (2009). Laser energy ( $4 \mathrm{~kJ}$ in a $1 \mathrm{~ns}$ pulse for this case) is delivered to an $820 \mu \mathrm{m}$ diameter (FWHM) spot on an ablator covering the low density foam part of the target (on the left of Fig. 2). In this way, a strong shock is launched into the low density foam such that the pressure gradient at the leading edge of the shock would essentially be at right angles to the density gradient at the interface of the two dissimilar materials thus maximizing $\nabla P \times \nabla \rho$. The interface between the two materials is perturbed by a sinusoidal contour with amplitude $(a=30 \mu \mathrm{m})$ and wavelength $(\lambda=400 \mu \mathrm{m})$ chosen such that a number of large vortices would develop nonlinear structure in the expected field of view during the experiment. The data from our initial experiments were largely consistent with expectations based upon two-dimensional (2D) simulation.

\subsection{Instability growth}

The images shown in Fig. 1 are simply converted into datum of vortex height versus time (Harding et al. 2009) that can be 

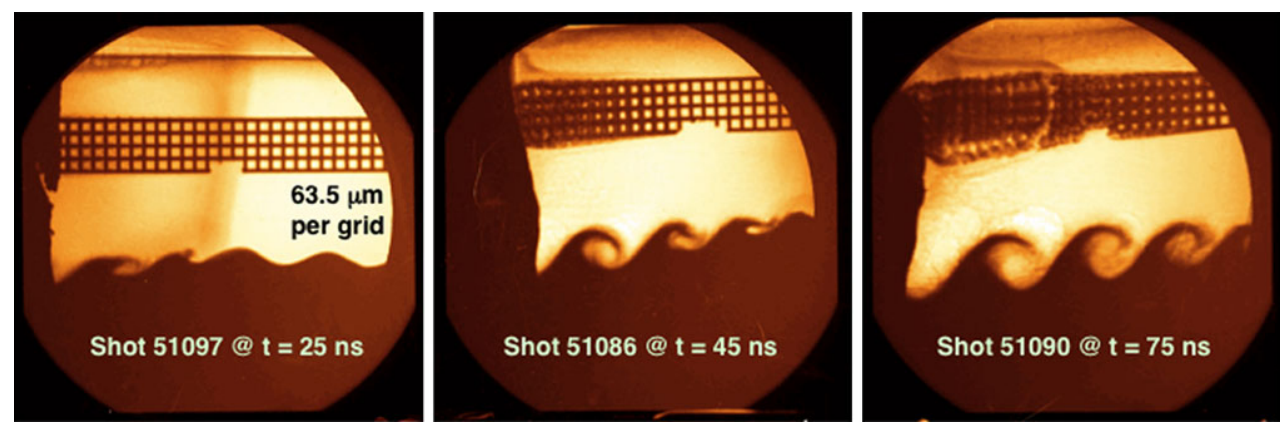

Fig. 1 From left to right, radiographic data from Omega shots 51097, 51086, and 51090 are shown. These three images show the time development of the $\mathrm{KH}$ instability at $25 \mathrm{~ns}, 45 \mathrm{~ns}$, and $75 \mathrm{~ns}$ respectively. In the left frame, the vorticity producing shock wave is visible in the low density $(100 \mathrm{mg} / \mathrm{cc})$ carbon foam. Wave crests begin to develop

compared with simulation and theory. In Fig. 3 an updated comparison of the vortex height data is shown against a revised simulation result and theory. The data shown in Fig. 3 are identical to those shown in Hurricane et al. (2009), Harding et al. (2009), but the simulation result shown here superceeds that presented previously. Here the simulation used to produce the data shown in Fig. 3 has been corrected to include the actual as-shot Be shock tube thickness of $500 \mu \mathrm{m}$ rather than the $200 \mu \mathrm{m}$ thickness used for the simulations shown in Hurricane et al. (2009), Harding et al. (2009) and a more accurate method of determining the vortex height from the simulation has also been used.

The vortex model theory shown in Fig. 3 comes from using the expression for the fluid circulation, $\Gamma$, derived in Hurricane (2008) (with values $P=1.62 \mathrm{Mbar}, \rho_{H}=1.43$ $\mathrm{g} / \mathrm{cc}, \rho_{L}=0.1 \mathrm{~g} / \mathrm{cc}$, and $\left.\gamma=5 / 3\right)$ in combination with the differential equations for the flow field

$$
\begin{aligned}
& \frac{d x}{d t}=\frac{\Gamma}{4 \lambda} \frac{\sinh \left(\frac{2 \pi y}{\lambda}\right)}{\cos ^{2}\left(\frac{\pi x}{\lambda}\right)+\sinh ^{2}\left(\frac{\pi y}{\lambda}\right)} ; \\
& \frac{d y}{d t}=\frac{\Gamma}{4 \lambda} \frac{\sin \left(\frac{2 \pi x}{\lambda}\right)}{\cos ^{2}\left(\frac{\pi x}{\lambda}\right)+\sinh ^{2}\left(\frac{\pi y}{\lambda}\right)}
\end{aligned}
$$

where these differential equations imply vortex growth up to a saturation of the vortex amplitude to a value of $y_{\text {max }}=\cosh ^{-1}(3) \lambda / 2 \pi \approx 0.281 \lambda$ (Hurricane 2008; Rikanati et al. 2003) the full vortex height then being $h_{\max }=$ $2 y_{\max }$. Since (2) trace out the trajectory that a massless particle would follow starting from some initial point $\left(x_{0}, y_{0}\right)$ at $t=0$, the full vortex height as plotted in Fig. 3 is then twice the value of the envelope of solutions to (2) using the $(x, y)$ locations that trace out the initial interface (see Fig. 4). An attempt to include the added complication of flow in the direction of vortex growth, due to the effect the transmitted shock, is shown in Fig. 3 as the stretched vortex model and is arrived at by adding a constant $y$-velocity of $2 \mu \mathrm{m} / \mathrm{ns}$ (from simulation) to the vortex model solution. immediately after passage of the shock wave and grow into fully developed vortices (middle frame). At late time (right frame), the spiral arms of the vortices appear to begin to diffuse away presumably the result of the onset of turbulence (figure adapted from Hurricane et al. 2009; Harding et al. 2009)

At late-time, the simulation and the stretched vortex model (which uses simulation derived values) both over predict the data. The simulation does exhibit the same change in growth rate at around $t=38 \mathrm{~ns}$ that the data show. Inspection of the simulation at $t=38 \mathrm{~ns}$ indicates that this is the time at which a shock traveling in the $-\hat{y}$ direction, that was reflected from the top of the Be shock tube, impacts the chain of vortices slightly compressing them.

The late-time over-prediction of the simulation is likely explained by the fact that the simulation is $2 \mathrm{D}$, while the target itself is 3D. That is in 2D, the simulation the post-shock expansion of the shock-tube would under-estimate the real decay in the post-shock flow that results from the shocktube expanding in 3D. Circumstantial evidence that supports this 3D shock-tube expansion hypothesis, is that the earlier 2D simulation Hurricane et al. (2009) that uses a thinner Be shock-tube wall thickness than the simulation presented here is closer to the data at late time. An actual 3D simulation would be necessary to fully prove this hypothesis and some effort in that direction is underway. Another possible reason for the over-prediction of the simulation is that the monotonic-Q (an artificial dissipation term needed to stabilize the simulation against shocks is known to over-deposit vorticity - a tensor- $\mathrm{Q}$ would be needed to alleviate this numeric problem.

\section{Second campaign of experiments}

A second series of experiments was performed at the Omega laser facility of the University of Rochester, in late 2009. In these experiments the density of the CRF foam was varied having two targets each of $50 \mathrm{mg} / \mathrm{cc}, 100 \mathrm{mg} / \mathrm{cc}$, and $200 \mathrm{mg} / \mathrm{cc}$. The purpose of the density variation was intended to study a cavitation-like behavior observed in the first series of experiments (see Hurricane et al. 2009), explore the density sensitivity of the instability growth, and obtain data on reproducibility of the vortex structures. 

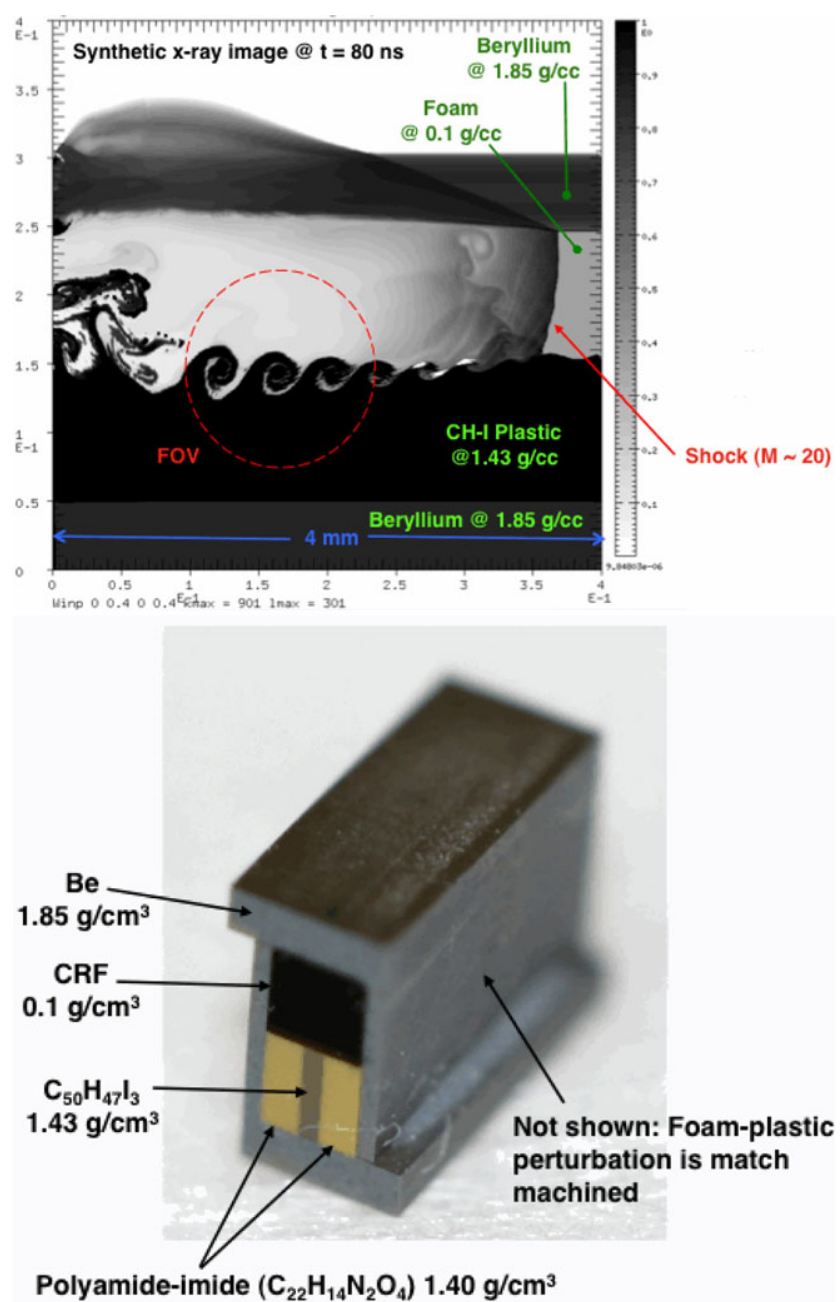

Fig. 2 Top: A simulated radiograph result of a simulation of the target performance is shown at $t=80 \mathrm{~ns}$. The materials that compose the target are annotated in the image. The field-of-view (FOV) accessible in the actual experiment is shown as the dashed red circle. The plasma conditions in the foam immediately behind the shock at $25 \mathrm{~ns}$ are $\rho=0.515 \mathrm{~g} / \mathrm{cc}, p=1.62 \mathrm{Mbars}, T_{\text {material }}=12 \mathrm{eV}$, and $v_{x}=3.55 \mathrm{~cm} / \mu \mathrm{s}$ (Hurricane et al. 2009). Bottom: an annotated picture of the actual target. Note that the Be tube sides are $200 \mu \mathrm{m}$ thick, while the top and bottom sections of the Be tube are $500 \mu \mathrm{m}$ thick

Results of the second set of experiments are shown in Fig. 5. As expected, the gross features of the first experiment are well duplicated. For the two good pairs of experiments in the series at 50 and $100 \mathrm{mg} / \mathrm{cc}$ respectively, many of the observed structures reproduce, one exception being the second largest vortex of shots 56127 and 56134. A quantitative comparison of the vortex growth versus time from these experiments will be forth-coming.

These later experiments appear to reveal more fine-scale structure than was observed in the original May 2008 series of experiments. This is largely due to the fact that in the first experimental series, two of the three images where at earlier stages in the instability evolution, while the later experiments were all imaged at late-time (with image times for

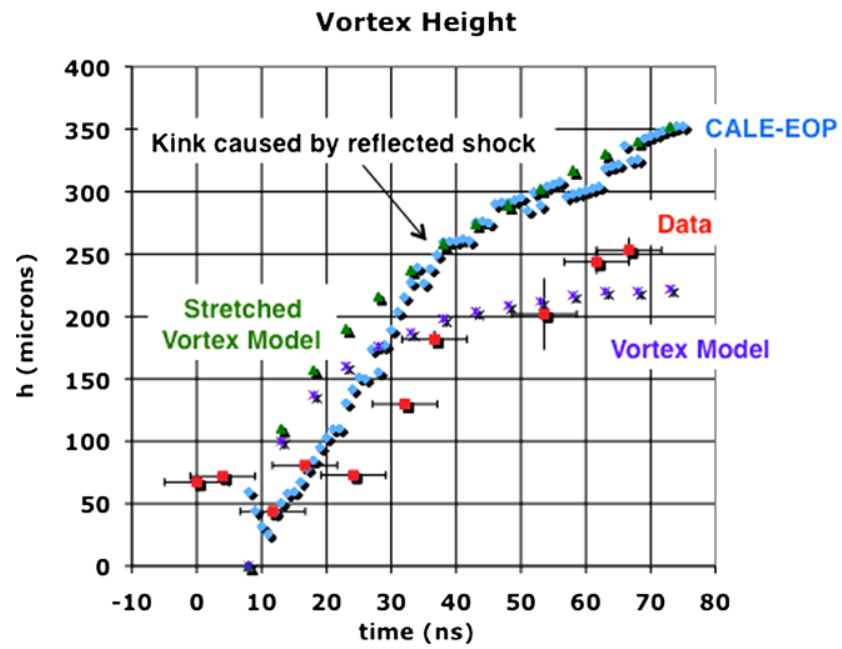

Fig. 3 Vortex height versus time is shown for the experiment (red squares), the simulation (blue diamonds), and theory (purple asterisks and green triangles). The data and simulation both show a period of post-shock amplitude compression between 10-15 ns and subsequent amplitude growth. A distinct change in growth-rate is seen around $38 \mathrm{~ns}$

\section{$h(t)$ shear layer height given by envelope}

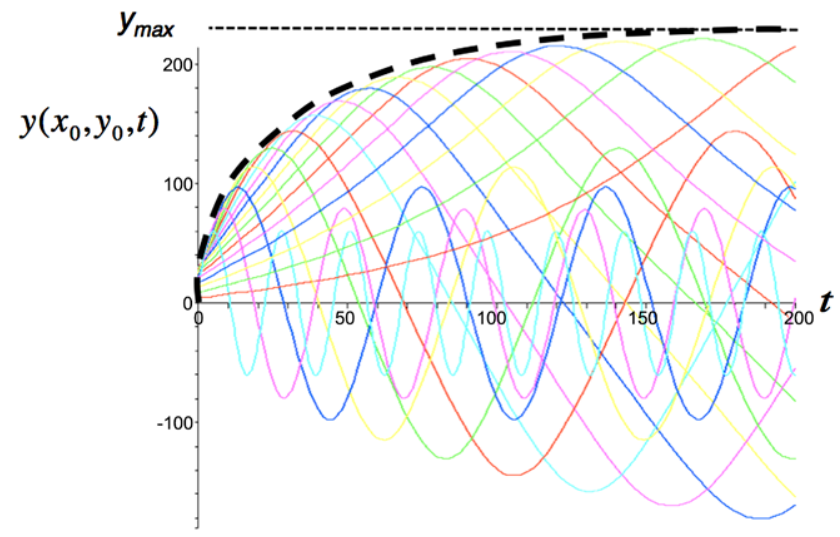

Max. vortex amplitude,

$y_{\max }=\cosh ^{-1}(3) \lambda / 2 \pi \approx 0.281 \lambda$

Fig. 4 A family of solutions to the vortex model is shown and the envelope of these solutions is given by the dashed curve

the different CRF densities scaled such that the blast-wave that deposits the vorticity is at the same location down the target in each case).

For the $100 \mathrm{mg} / \mathrm{cc}$ CRF foam cases (shots 51086, 51090, and 56134), comparing images of early-time instability to later time, a mixing transition is strongly suggested since coherent structures of the earlier time vortex (e.g. the vortex spiral-arm) is breaking up and fading away (see Fig. 6). Moreover the distinct boundary between the $\mathrm{CH}$-Iodine plastic and CRF foam, seen in 51086 appears to become fuzzy in later time images (51090 and 56134). A mixing transition is consistent with the calculation of two important 
Fig. 5 Radiographic images from the second series of experiments are shown and labeled by shot number
Fig. 6 Close-up images of the radiographic images from shots using the $100 \mathrm{mg} / \mathrm{cc}$ foam are suggestive of a transition from a smooth flow to a more turbulent flow
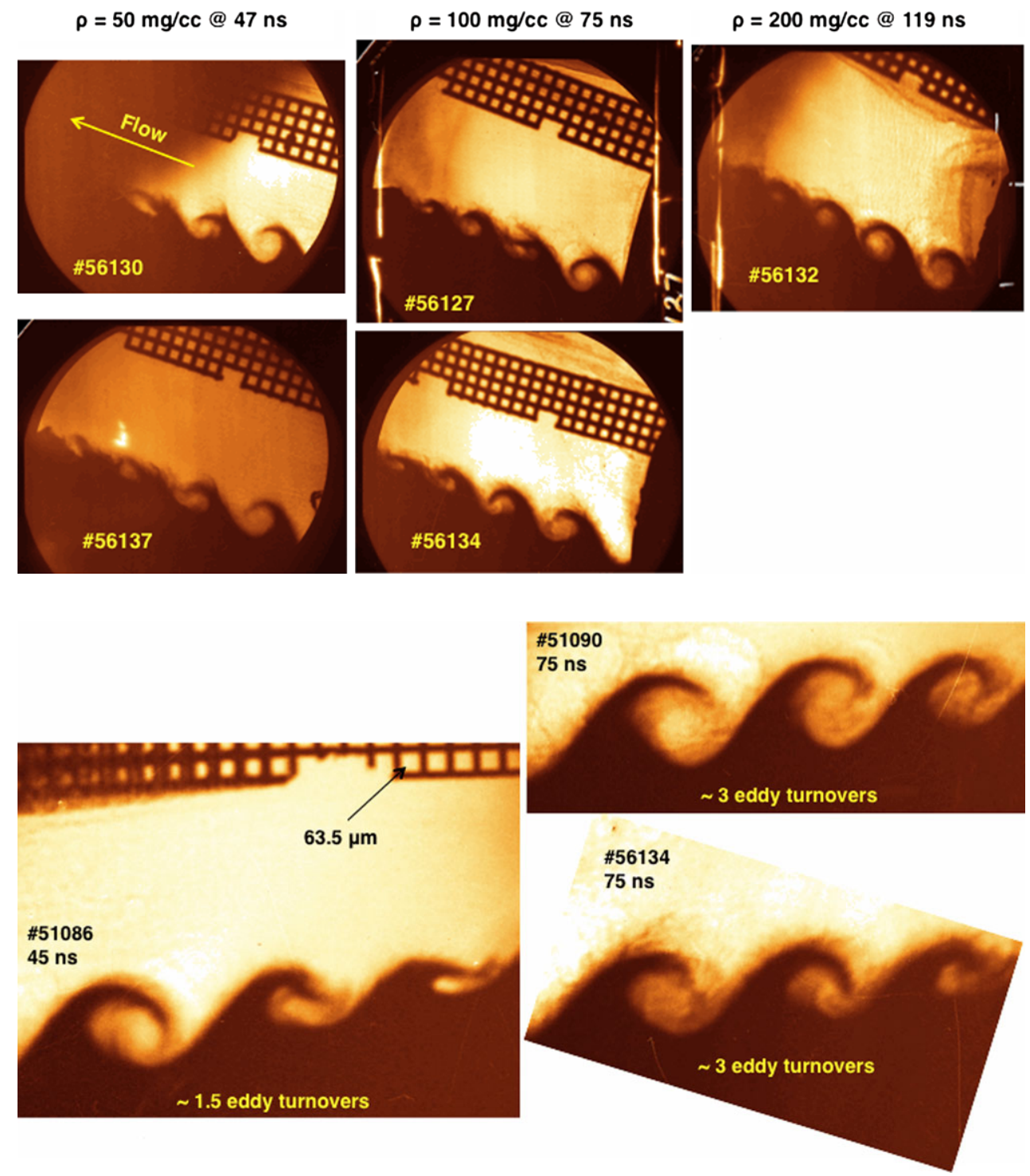

length-scales (see Robey 2004; Zhou 2007): the LiepmannTaylor scale and inner-viscous scale which calculate to be $2 \mu \mathrm{m}$ and $0.65 \mu \mathrm{m}$ respectively (based upon a viscosity of $v \sim 0.014 \mathrm{~cm}^{2} / \mathrm{s}$ inferred from the data using Clérouin et al. 1998). These scales, define the "inertial range," within which the Kolmogorov turbulent cascade can be active. Above the Liepmann-Taylor scale imposed forcing scales of the flow dominate, while below the inner-viscous scale viscous damping dominates the flow. More work will be required to conclusively establish a transition to turbulence.

As for "bubble" structures, the two $50 \mathrm{mg} / \mathrm{cc}$ density foam experiment images (shots 56130 and 56137) showed no evidence of bubble structures above the vortex line. Only one of the $100 \mathrm{mg} / \mathrm{cc}$ experiment images showed a bubble (56127), while the other image (from shot 56134) was too overexposed to discern any bubbles. The $200 \mathrm{mg} / \mathrm{cc}$ experiment (shot 56132) also showed bubbles. However, in no cases were the bubbles observed in this series of experiments as clear and regular as in the original experiment series. Clearly, this inconsistent appearance of these bubble structures makes discerning their nature more difficult.

\section{Conclusion}

While some features seen in our HEDP KH experiment are yet to be fully explained, we have demonstrated to viability of the target design concept and demonstrated that high quality data on a $\mathrm{KH}$ unstable shear layer can be obtained providing a unique test of simulation and theory. Our simulation and theory are in fair agreement with the data obtained from this experiment with regard to the overall height of the vortex layer, but more detailed structures in the flow were not predicted by the simulation even though we knew the initial conditions very well. 
Further extensions of this platform to study multi-mode $\mathrm{KH}$ under HEDP conditions is easily accomplished by judicious choice of initial interface perturbation. Another interesting possible experiment would be to study the expected supersonic stabilization of KH (e.g. the Mach number $M>2 \sqrt{2}$ condition of Landau, 1944). Future experiments on NIF could create sustained steady flows with higher Reynolds numbers and use larger targets (for better diagnosis) both of which would enhance the usefulness of this platform.

This platform could enable validation of astrophysics codes and the physics of this platform is potentially directly relevant to the interaction of supernova with nearby protoplanetary disks, astrophysical jets, and stellar wind-cloud interactions. A recent astronomical observation of KelvinHelmholtz instability occurring during the expansion of hot, ionized nebular gas sweeping over denser gas in the Orion molecular cloud is a striking example of the role played by $\mathrm{KH}$ in astrophysics Berne et al. (2010).

Acknowledgements This work has benefitted from the support of Dr. Alan Wan and Dr. Charlie Verdon. Thanks to Prof. R. Paul Drake for technical comments and discussion of this work. This work was performed under the auspices of the U.S. Department of Energy
Lawrence Livermore National Laboratory under contract No. DEAC52-07NA27344 and support by a National Laser User Facilities Grant.

Open Access This article is distributed under the terms of the Creative Commons Attribution Noncommercial License which permits any noncommercial use, distribution, and reproduction in any medium, provided the original author(s) and source are credited.

\section{References}

Berne, O., Marcelino, N., Cernicharo, J.: Nat. Lett. 466, 947 (2010)

Clérouin, J.G., Cherfi, M.H., Zérah, G.: Europhys. Lett. 42, 37 (1998)

Harding, E.C., Hansen, J.F., Hurricane, O.A., Drake, R.P., Robey, H.F., Kuranz, C.C., Remington, B.A., Bono, M.J., Grosskopf, M.J., Gillespie, R.S.: Phys. Rev. Lett. 103, 045005 (2009)

Hurricane, O.A.: High Energy Den. Phys. doi:10.1016/j.hedp.2008. 02002 (2008)

Hurricane, O.A., Hansen, J.F., Robey, H.F., Remington, B.A., Bono, M.J., Harding, E.C., Drake, R.P., Kuranz, C.C.: Phys. Plasmas 16, 056305 (2009)

Rikanati, A., Alon, U., Shvarts, D.: Phys. Fluids 15, 3776 (2003)

Robey, H.F.: Phys. Plasmas 11, 4123 (2004)

Zhou, Y.: Phys. Plasmas 14, 082701 (2007) 